\title{
Experimental Study on the Effects of X-ray Irradiation on the Pancreatic Cells.
}

\author{
$\mathrm{X}$ 線照射が膵細胞に及ぼす影響に関する実験的研究。
}

\author{
Junji HATANAKA 畑中淳治.
}

(Received september $26,1960$.

It is well known that the most frequent symptom of radiotoxemia is concerned with the digestive system and that the symptom does not accompany any histological changes unless an extremely large dose is given. KUSUMOTO proved that the secretory activity of the gastric peptic cells of rats was lowered positively after the irradiation of $500 \mathrm{r}$ on the epigastrium and on the hypogastrium-thighs region regardless of the area of the irradiation, and that the secretion of the histamine-like hormone productin (FUJIE) from the gastric surface cells which promotes the secretory activity of the peptic cells was not lowered to any remarkable degree. He further injected subcutaneously Histamine $2 \mathrm{HCl}$ prior to the X-ray irradiation and found that the action of the discharged productin was inhibited by a certain substance which might be produced in the tissue irradiated.

The secretory activity of the pancreatic cells, especially the production of zymogen granules, is said to be promoted by the productin as well (FUJIE). The author attempted to study the effects of the X-ray irradiation on the pancreatic cells so that the action of the X-ray irradiation on the productin behaviour might be made clearer.

\section{Materials and Methods.}

Adult and male albino rats accustomed to a regular feeding of Mc COLLUM's artificial diet were used, and all the animals were unfed for $24 \mathrm{hrs}$. before the experiment.

Series A. Control.

An artificial diet was given. Samples were taken before and at periods of $\frac{1}{2}, 1$, 2, 3, 6 hrs. after the diet.

Series $B$. For the study on the effects of X-ray irradiation.

1. A single dose $500 \mathrm{r}$ was given on the epigastrium, an hour later the diet was given. Samples were taken at the same periods as in the controls.

2. A daily dose $100 \mathrm{r}$ was given on the epigastrium for 5 days before every feeding. Samples were taken before and after the last diet at the same periods as above.

3. A single dose $500 \mathrm{r}$ was given on the hypogastrium and the thigh, an hour later the diet was given.

4. A daily dose $100 \mathrm{r}$ was given for 5 days on the hypogastrium-thighs region, an hour later the diet was given.

Series $C$. For study on the protective effects of some chemical agents against 
$\mathrm{X}$-ray injuries.

1. Prior to the irradiation of $500 \mathrm{r}$ on the epigastrium and to the diet, Pereston- $\mathrm{N}$ (polyvinylpyrrolidon) $10 \mathrm{cc} / \mathrm{kg}$ weight was injected into the abdominal cavity. Viz. 5 min. after the injection, X-ray was irradiated and, an hour after the irradiation, the diet was given. Samples were taken before and at periods of $\frac{1}{2}, 1,2,3$, $6 \mathrm{hrs}$. after the diet.

2. Thioctic acid $12.5 \mathrm{mg} / \mathrm{kg}$ weight was injected into the abdominal cavity prior to the administration of $\mathrm{X}$-ray and the diet.

3. 5-oxy-anthranilic acid $10 \mathrm{mg} / \mathrm{kg}$ weight and $100 \mathrm{mg} / \mathrm{kg}$ was injected into the abdominal cavity before the irradiation and the diet.

Throughout these experiments, the X-ray irradiation was conditioned as follows: Tube voltage $180 \mathrm{KVp}$, tube current $15 \mathrm{~mA}$, filter $1.0 \mathrm{~mm} \mathrm{Cu}$ and $1.5 \mathrm{~mm} \mathrm{Al}$, focal skin distance $400 \mathrm{~mm}$ and rate dose $52.9 \mathrm{r} / \mathrm{min}$. During the irradiation, the animals were laid on their backs and enclosed in a $3 \mathrm{~mm}$ thick leaden cover with a hole of $35 \times 50 \mathrm{~mm}$. It was so arranged that the hole was over the epigastrium or the hypogastrium and the thigh.

To take samples, the thorax was opened under deep ether narcosis and LUNA's liquid was injected into all arteries through the left heart ventricle for the vital fixation. Small pieces were taken from the pancreas and they were placed in ZENKER's and KOLSTER's liquid for the post fixation. Paraffin sections of $7 \mu$ were made from the pancreas fixed by ZENKER's liquid and of $4 \mu$ were made from that fixed by KOLSTER's liquid. The formor sections were stained by haematoxylin-eosin and the latter were by HEIDENHAIN's iron haematoxylin.

\section{Observations.}

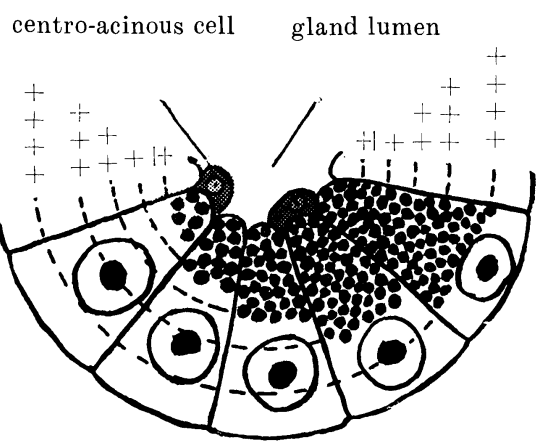

Fig. 1. Diagram of the quantity of the zymogen granules in the pancreatic cells.

massive, blackened etc., concerning which see Fig. 2). From these composite observations the productive and the discharging activities of zymogen granules in the cells were determined. a) Cytological observations.

The secretory activity of the pancreatic cells was presumed from the minute structure of the cells. The author described 1. quantity of zymogen granules in the cell $(-, \pm,+,++,+++$ etc., concerning which see Fig. 1), 2. distribution of same (loose, normal, dense etc.), 3. irregularity in their size (little, a little, large etc.), 4. the form of plastosomes (pls.) and 5. the aspect of the gland lumen (empty, straggling,

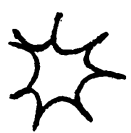

empty

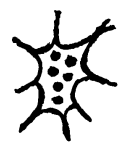

straggling

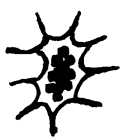

massive

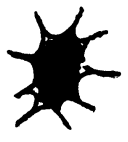

blackened
Fig. 2. Standard figures of the aspect of the pancreatic gland lumen. 
The results obtained were tabulated in Table $1-3$.

Table 1. Controlling and epigastrium irradiation.

\begin{tabular}{|c|c|c|c|c|c|c|c|c|}
\hline \multirow{2}{*}{ 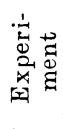 } & \multirow{2}{*}{ Hrs. } & \multicolumn{3}{|c|}{ Zymogen granules } & \multirow{2}{*}{$\begin{array}{l}\text { Form of } \\
\text { pls. }\end{array}$} & \multirow{2}{*}{$\begin{array}{l}\text { Production } \\
\text { to be } \\
\text { considered }\end{array}$} & \multirow{2}{*}{$\begin{array}{l}\text { Gland } \\
\text { lumen }\end{array}$} & \multirow{2}{*}{$\begin{array}{l}\text { Discharge } \\
\text { to be } \\
\text { considered }\end{array}$} \\
\hline & & Quantity & $\begin{array}{l}\text { Distribu- } \\
\text { tion }\end{array}$ & $\begin{array}{c}\text { Irregulari- } \\
\text { ty in size }\end{array}$ & & & & \\
\hline \multirow{6}{*}{ 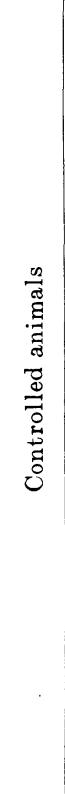 } & $\begin{array}{l}\mathrm{Be}- \\
\text { fore }\end{array}$ & $++\sim+++$ & loose & a little & $\begin{array}{l}\text { long rod, } \\
\text { short rod, } \\
\text { filament, } \\
\text { rosary form }\end{array}$ & weakest & $\begin{array}{c}\text { empty, } \\
\text { straggling }\end{array}$ & weakest \\
\hline & $\frac{1}{2}$ & $\pm \sim+++(+)$ & $\begin{array}{c}\text { loose } \\
\text { n. }\end{array}$ & large & $\begin{array}{l}\text { short rod, } \\
\text { guitar form, } \\
\text { rosary form, } \\
\text { long rod }\end{array}$ & middle & $\begin{array}{l}\text { straggling. } \\
\text { empty, } \\
\text { massive }\end{array}$ & middle \\
\hline & 1 & $\pm \sim++$ & loose & large & $\begin{array}{l}\text { guitar form, } \\
\text { short rod, } \\
\text { supra-short } \\
\text { rod, rosary } \\
\text { form }\end{array}$ & middle & $\begin{array}{l}\text { straggling, } \\
\text { massive, } \\
\text { empty, } \\
\text { blackened }\end{array}$ & strong \\
\hline & 2 & $-\sim++$ & loose & large & $\begin{array}{l}\text { guitar form, } \\
\text { supra-short } \\
\text { rod, short } \\
\text { rod, rosary } \\
\text { form }\end{array}$ & middle & $\begin{array}{l}\text { straggling, } \\
\text { massive, } \\
\text { empty }\end{array}$ & middle \\
\hline & 3 & $\pm \sim++$ & $\begin{array}{c}\text { loose } \\
\text { n. }\end{array}$ & a little & $\begin{array}{l}\text { short rod, } \\
\text { guitar form, } \\
\text { rosary form, } \\
\text { long rod }\end{array}$ & $\left.\right|_{\text {weak }} ^{\text {middle }}$ & $\begin{array}{c}\text { straggling, } \\
\text { empty, } \\
\text { massive }\end{array}$ & $\begin{array}{c}\text { middle } \\
\text { weak }\end{array}$ \\
\hline & 6 & $\begin{array}{r}+(+) \sim \\
+++\end{array}$ & $\begin{array}{c}\text { loose } \\
\text { n. }\end{array}$ & a little & $\begin{array}{l}\text { short rod, } \\
\text { rosary form, } \\
\text { guitar form, } \\
\text { long rod }\end{array}$ & weak & $\begin{array}{c}\text { straggling, } \\
\text { empty }\end{array}$ & weak \\
\hline \multirow{6}{*}{ 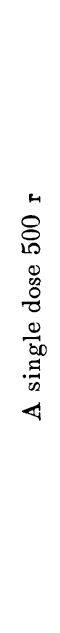 } & $\begin{array}{l}\text { Be- } \\
\text { fore }\end{array}$ & $\begin{array}{l}+(+) \sim \\
+++\end{array}$ & $\stackrel{\text { n. }}{!}$ & little & $\begin{array}{l}\text { long rod, } \\
\text { short rod, } \\
\text { rose,ry form }\end{array}$ & $\begin{array}{c}\text { weakest } \\
\text { ! } \\
\text { weak }\end{array}$ & $\begin{array}{c}\text { empty, } \\
\text { straggling, } \\
\text { massive }\end{array}$ & weak \\
\hline & $\frac{1}{2}$ & $+\sim+t+$ & n. & a little & $\begin{array}{l}\text { short rod, } \\
\text { long rod, } \\
\text { guitar form, } \\
\text { rosary form }\end{array}$ & weak & $\begin{array}{c}\text { straggling, } \\
\text { empty }\end{array}$ & weak \\
\hline & 1 & $\pm \sim++$ & $\begin{array}{c}\text { loose } \\
\text { n. }\end{array}$ & little & $\begin{array}{l}\text { short rod, } \\
\text { long rod, } \\
\text { rosary form }\end{array}$ & weak & $\begin{array}{l}\text { straggling, } \\
\text { empty, } \\
\text { massive }\end{array}$ & $\begin{array}{c}\text { middle } \\
\text { weak }\end{array}$ \\
\hline & 2 & $+\sim+t+$ & $\mathrm{n}$. & a little & $\begin{array}{l}\text { long rod, } \\
\text { short rod, } \\
\text { rosary form }\end{array}$ & weak & $\begin{array}{c}\text { empty, } \\
\text { straggling, } \\
\text { massive }\end{array}$ & $\left.\right|_{\text {middle }} ^{\text {weak }}$ \\
\hline & 3 & $+\sim++(+)$ & $\stackrel{\text { n. }}{\mid}$ & a little & $\begin{array}{l}\text { long rod, } \\
\text { short rod, } \\
\text { guitar form }\end{array}$ & weak & $\begin{array}{c}\text { straggling, } \\
\text { empty }\end{array}$ & weak \\
\hline & 6 & $\pm \sim++(+)$ & $\stackrel{\text { n. }}{i}$ & little & $\begin{array}{l}\text { long rod, } \\
\text { short rod, } \\
\text { rosary form }\end{array}$ & weak & $\begin{array}{c}\text { straggling, } \\
\text { massive, } \\
\text { empty }\end{array}$ & middle \\
\hline
\end{tabular}




\begin{tabular}{|c|c|c|c|c|c|c|c|c|}
\hline & $\begin{array}{l}\text { Be- } \\
\text { fore }\end{array}$ & $+\sim+++$ & loose & little & $\begin{array}{l}\text { long rod, } \\
\text { filament, } \\
\text { short rod }\end{array}$ & weakest & $\begin{array}{c}\text { empty, } \\
\text { straggling, } \\
\text { (massive) }\end{array}$ & $\begin{array}{c}\text { weak } \\
\text { (middle) }\end{array}$ \\
\hline$\stackrel{n}{\infty}$ & $\frac{1}{2}$ & $+\sim+++$ & $\left.\right|_{\text {loose }} ^{\text {n. }}$ & a little & $\begin{array}{l}\text { short rod, } \\
\text { long rod, } \\
\text { rosary form }\end{array}$ & weak & $\begin{array}{c}\text { empty, } \\
\text { straggling, } \\
\text { (massive) }\end{array}$ & (middle) \\
\hline $\begin{array}{l}0 \\
10 \\
10\end{array}$ & 1 & $+\sim+++$ & $\left.\right|_{\text {loose }} ^{\text {n. }}$ & a little & $\begin{array}{l}\text { short rod, } \\
\text { long rod, } \\
\text { guitar form }\end{array}$ & weak & $\begin{array}{l}\text { straggling, } \\
\text { empty }\end{array}$ & weak \\
\hline $\begin{array}{l}8 \\
\stackrel{8}{-1} \\
\stackrel{2}{7}\end{array}$ & 2 & $\begin{array}{r}+(+) \sim \\
+++\end{array}$ & $\left.\right|_{\text {loose }} ^{\text {n. }}$ & a little & $\begin{array}{l}\text { long rod, } \\
\text { short rod, } \\
\text { guitar form }\end{array}$ & weak & $\begin{array}{c}\text { empty, } \\
\text { straggling }\end{array}$ & weakest \\
\hline$A$ & 3 & $+\sim+++$ & $\left.\right|_{\text {loose }} ^{\text {n. }}$ & a little & $\begin{array}{l}\text { short rod, } \\
\text { long rod, } \\
\text { rosary form }\end{array}$ & weak & $\begin{array}{l}\text { straggling, } \\
\text { empty }\end{array}$ & weak \\
\hline & 6 & $\pm \sim++(+)$ & loose & a little & $\begin{array}{l}\text { long rod, } \\
\text { short rod }\end{array}$ & weakest & $\begin{array}{l}\text { empty, } \\
\text { straggling, } \\
\text { (massive) }\end{array}$ & $\begin{array}{c}\text { weak } \\
\text { (middle) }\end{array}$ \\
\hline
\end{tabular}

Table 2. Hypogastrium-thigh irradiation.

\begin{tabular}{|c|c|c|c|c|c|c|c|c|}
\hline \multirow{2}{*}{ 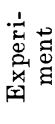 } & \multirow{2}{*}{ Hrs. } & \multicolumn{3}{|c|}{ Zymogen granules } & \multirow{2}{*}{$\begin{array}{l}\text { Form of } \\
\text { pls. }\end{array}$} & \multirow{2}{*}{$\begin{array}{l}\text { Production } \\
\text { to be } \\
\text { considered }\end{array}$} & \multirow{2}{*}{$\begin{array}{l}\text { Gland } \\
\text { lumen }\end{array}$} & \multirow{2}{*}{$\begin{array}{l}\text { Discharge } \\
\text { to be } \\
\text { considered }\end{array}$} \\
\hline & & Quantity & $\begin{array}{c}\text { Distribu- } \\
\text { tion }\end{array}$ & $\begin{array}{l}\text { Irregulari- } \\
\text { ty in size }\end{array}$ & & & & \\
\hline \multirow{6}{*}{ 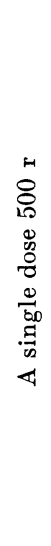 } & $\begin{array}{c}\mathrm{Be}- \\
\text { fore }\end{array}$ & $+\sim+++$ & n. & a little & $\begin{array}{l}\text { long rod, } \\
\text { short rod, } \\
\text { rosary form }\end{array}$ & weak & $\begin{array}{c}\text { empty, } \\
\text { straggling, } \\
\text { (massive) }\end{array}$ & weak \\
\hline & $\frac{1}{2}$ & $+\sim+++$ & n. & a little & $\begin{array}{l}\text { short rod, } \\
\text { rosary form, } \\
\text { long rod }\end{array}$ & (middle) & $\begin{array}{c}\text { straggling, } \\
\text { empty, } \\
\text { massive }\end{array}$ & $\begin{array}{c}\text { middle } \\
! \\
\text { weak }\end{array}$ \\
\hline & 1 & $+\sim++(+)$ & $\mathrm{n}$. & a little & $\begin{array}{l}\text { short rod, } \\
\text { long rod, } \\
\text { rosary form }\end{array}$ & weak & \begin{tabular}{|} 
straggling, \\
massive, \\
empty
\end{tabular} & middle \\
\hline & 2 & $\pm \sim+++$ & 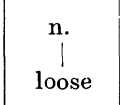 & $\begin{array}{l}\text { a little } \\
\text { large }\end{array}$ & $\begin{array}{l}\text { short rod, } \\
\text { rosary form, } \\
\text { guitar form }\end{array}$ & middle & $\begin{array}{l}\text { stragglign, } \\
\text { empty, } \\
\text { massive }\end{array}$ & $\left.\right|_{\text {weak }} ^{\text {middle }}$ \\
\hline & 3 & $+\sim++(+)$ & n. & a little & $\begin{array}{l}\text { short rod, } \\
\text { long rod, } \\
\text { rosary form }\end{array}$ & weak & $\begin{array}{c}\text { straggling, } \\
\text { empty, } \\
\text { massive }\end{array}$ & $\left.\right|_{\text {weak }} ^{\text {middle }}$ \\
\hline & 6 & $+\sim+++$ & n. & a little & $\begin{array}{l}\text { long rod, } \\
\text { short rod }\end{array}$ & weakest & $\begin{array}{c}\text { empty, } \\
\text { straggling }\end{array}$ & weakest \\
\hline & $\begin{array}{l}\text { Be- } \\
\text { fore }\end{array}$ & $+\sim+t+$ & $\stackrel{\mathrm{n} .}{i}$ & a little & $\begin{array}{l}\text { long rod, } \\
\text { short rod, } \\
\text { guitar form }\end{array}$ & weak & $\begin{array}{c}\text { empty, } \\
\text { straggling, } \\
\text { massive }\end{array}$ & weak \\
\hline$\stackrel{\infty}{\infty}$ & $\frac{1}{2}$ & $\pm \sim++$ & $\mathrm{n}$. & a little & $\begin{array}{l}\text { short rod, } \\
\text { long rod, } \\
\text { rosary form }\end{array}$ & weak & $\begin{array}{c}\text { straggling, } \\
\text { massive, } \\
\text { empty }\end{array}$ & middle \\
\hline $\begin{array}{l}\text { Iึ } \\
\text { in } \\
8\end{array}$ & 1 & $+\sim+++$ & $\stackrel{\text { n. }}{i}$ & a little & $\begin{array}{l}\text { short rod, } \\
\text { guitar form, } \\
\text { rosary form, } \\
\text { long rod }\end{array}$ & middle & $\begin{array}{c}\text { straggling, } \\
\text { empty }\end{array}$ & weak \\
\hline 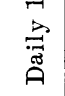 & 2 & $+\sim++(+)$ & n. & a little & $\begin{array}{l}\text { short rod, } \\
\text { long rod, } \\
\text { rosary form }\end{array}$ & weak & $\begin{array}{l}\text { straggling, } \\
\text { empty, } \\
\text { massive }\end{array}$ & $\begin{array}{c}\text { middle } \\
\text { weak }\end{array}$ \\
\hline
\end{tabular}


Experimental Study on the Effects of X-ray Irradiation on the Pancreatic Cells. 121

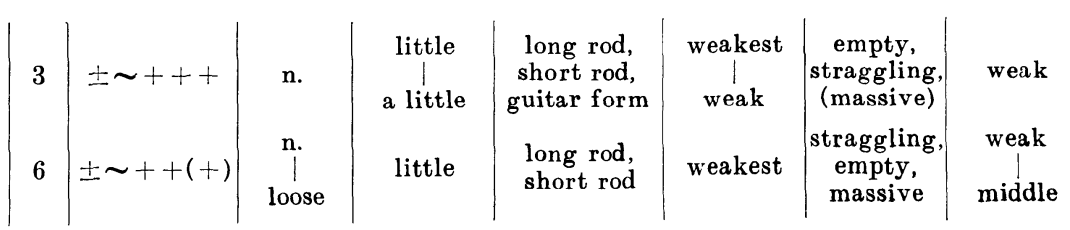

Table 3. Injection of protective chemical agents against the $\mathrm{X}$-ray injuries prior to the $500 \mathrm{r}$ irradiation on the epigastrium.

\begin{tabular}{|c|c|c|c|c|c|c|c|c|}
\hline \multirow{2}{*}{ 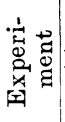 } & \multirow{2}{*}{ Hrs. } & \multicolumn{3}{|c|}{ Zymogen granules } & \multirow{2}{*}{$\begin{array}{l}\text { Form of } \\
\text { pls. }\end{array}$} & \multirow{2}{*}{$\begin{array}{l}\text { Production } \\
\text { to be } \\
\text { considered }\end{array}$} & \multirow{2}{*}{$\begin{array}{l}\text { Gland } \\
\text { lumen }\end{array}$} & \multirow{2}{*}{$\begin{array}{l}\text { Discharge } \\
\text { to be } \\
\text { consider ed }\end{array}$} \\
\hline & & Quantity & $\begin{array}{c}\text { Distribu- } \\
\text { tion }\end{array}$ & $\begin{array}{c}\text { Irregulari- } \\
\text { ty in size }\end{array}$ & & & & \\
\hline \multirow{6}{*}{ 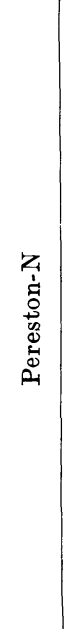 } & $\begin{array}{l}\text { Be- } \\
\text { fore }\end{array}$ & $\pm \sim++$ & $\mathrm{n}$. & little & $\begin{array}{c}\text { thick } \\
\text { long rod, } \\
\text { filanent, } \\
\text { short rod }\end{array}$ & weak & $\begin{array}{c}\text { empty, } \\
\text { straggling, } \\
\text { massive }\end{array}$ & meak \\
\hline & $\frac{1}{2}$ & $+\sim+++$ & n. & a little & $\begin{array}{l}\text { short rod, } \\
\text { rosary form, } \\
\text { long rod }\end{array}$ & $\left.\right|_{\text {middle }} ^{\text {weak }}$ & $\begin{array}{c}\text { empty, } \\
\text { straggling, } \\
\text { massive }\end{array}$ & middle \\
\hline & 1 & $\pm \sim++$ & n. & little & $\begin{array}{l}\text { long rod, } \\
\text { short rod }\end{array}$ & weakest & $\begin{array}{c}\text { straggling } \\
\text { empty }\end{array}$ & weak \\
\hline & 2 & $+\sim++(+)$ & loose & large & $\begin{array}{l}\text { guitar form, } \\
\text { short rod, } \\
\text { rosary form }\end{array}$ & middle & $\begin{array}{l}\text { straggling, } \\
\text { empty }\end{array}$ & weak \\
\hline & 3 & $+\sim+++$ & n. & a little & $\begin{array}{l}\text { short rod, } \\
\text { guitar form, } \\
\text { rosary form, } \\
\text { long rod }\end{array}$ & $\begin{array}{c}\text { middle } \\
\text { weak }\end{array}$ & $\begin{array}{c}\text { empty, } \\
\text { straggling }\end{array}$ & weakest \\
\hline & 6 & $\pm \sim++$ & loose & little & $\begin{array}{l}\text { long rod, } \\
\text { rosary form, } \\
\text { short rod }\end{array}$ & weak & $\begin{array}{c}\text { straggling, } \\
\text { blackened, } \\
\text { empty, } \\
\text { massive }\end{array}$ & $\underset{\text { strong }}{\text { middle }}$ \\
\hline \multirow{6}{*}{ 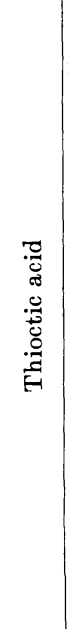 } & $\begin{array}{l}\mathrm{Be}- \\
\text { fore }\end{array}$ & $+\sim+++$ & loose & a little & $\begin{array}{l}\text { long rod, } \\
\text { short rod }\end{array}$ & weakest & $\begin{array}{c}\text { straggling, } \\
\text { empty, } \\
\text { massive }\end{array}$ & $\begin{array}{c}\text { weak } \\
\text { middle }\end{array}$ \\
\hline & $\frac{1}{2}$ & $+\sim+++$ & loose & a little & $\begin{array}{l}\text { short rod, } \\
\text { long rod, } \\
\text { rosary form }\end{array}$ & weak & $\begin{array}{c}\text { empty, } \\
\text { straggling, } \\
\text { (massive) }\end{array}$ & (middle) \\
\hline & 1 & $-\sim++$ & n. & large & $\begin{array}{l}\text { short rod, } \\
\text { guitar form, } \\
\text { supra-short } \\
\text { rod, long } \\
\text { rod }\end{array}$ & middle & $\begin{array}{c}\text { straggling, } \\
\text { massive, } \\
\text { blackened, } \\
\text { empty }\end{array}$ & strong \\
\hline & 2 & $\pm \sim++$ & n. & a little & $\begin{array}{l}\text { short rod, } \\
\text { guitar form, } \\
\text { rosary form, } \\
\text { long rod }\end{array}$ & $\begin{array}{c}\text { middle } \\
\text { | } \\
\text { weak }\end{array}$ & $\mid \begin{array}{c}\text { straggling, } \\
\text { empty, } \\
\text { massive, } \\
\text { (blackened) }\end{array}$ & $\underset{\text { (strong) }}{\stackrel{\mid}{\text { middle }}}$ \\
\hline & 3 & $\pm \sim++(+)$ & n. & little & $\begin{array}{l}\text { long rod, } \\
\text { short rod, } \\
\text { guitar form }\end{array}$ & weak & $\begin{array}{c}\text { straggling } \\
\text { empty, } \\
\text { (massive) }\end{array}$ & (middle) \\
\hline & 6 & $+\sim+++$ & loose & little & $\begin{array}{l}\text { long rod, } \\
\text { short rod }\end{array}$ & weakest & $\begin{array}{c}\text { empty, } \\
\text { straggling }\end{array}$ & weakest \\
\hline
\end{tabular}




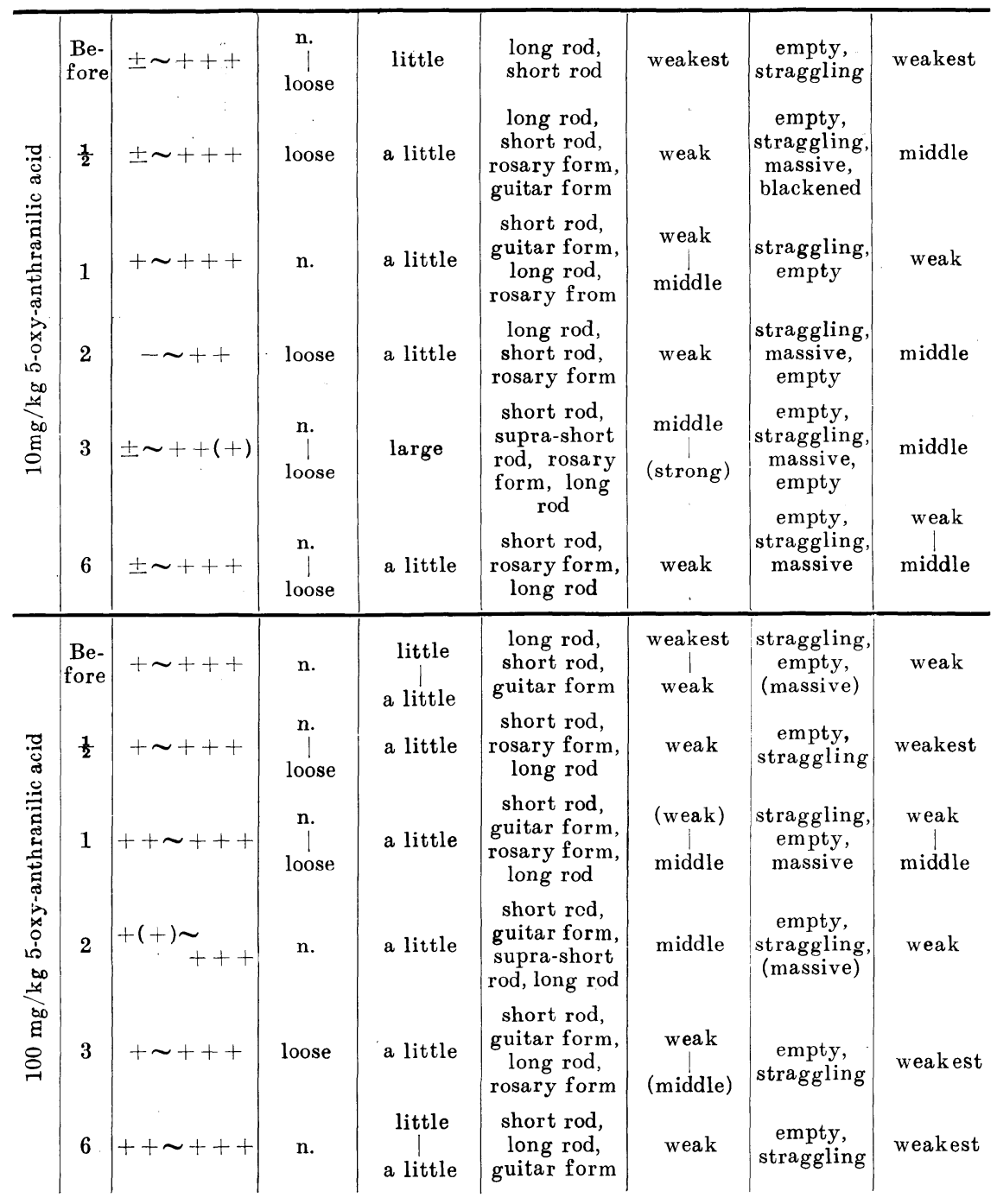

\section{b) Histological observations.}

No histological changes can be found in the pancreas. However, the number of the binucleate cells seems to be very different according to the different cases. The author counted the binucleate cells in the sectioned specimens of $7 \mu$ stained by haematoxylin-eosin, and obtained the results shown in Table 4 . This number must be smaller than where crushed pancreas was used, because it was proved by WADA in case of liver that the number of the binucleate cells counted in the sectioned specimens is only $47.6 \%$ of that counted in the crushed specimens. Anyhow, the differences in each case will be seen in the table. 
Table 4. Number of the binucleate cells in 1000 pancreatic cells.

\begin{tabular}{|c|c|c|c|c|c|c|c|c|c|}
\hline \multirow{2}{*}{ Hrs. } & \multicolumn{2}{|c|}{$\begin{array}{l}\text { Irradiation of } \\
\text { the epigastrium }\end{array}$} & \multicolumn{2}{|c|}{$\begin{array}{c}\text { Irradiation of the } \\
\text { hypogastrium-thigh }\end{array}$} & \multicolumn{4}{|c|}{$\begin{array}{c}500 \mathrm{r} \text { irradiation of the } \\
\text { epigastrium }\end{array}$} & \multirow{2}{*}{ o } \\
\hline & $500 \mathrm{r}$ & $\begin{array}{r}100 \mathrm{r} \\
\times 5\end{array}$ & $500 \mathrm{r}$ & $\begin{array}{r}100 \mathrm{r} \\
\times 5\end{array}$ & $\begin{array}{l}\text { Perest.- } \\
\quad \mathrm{N}\end{array}$ & $\begin{array}{l}\text { Thioct. } \\
\text { acid }\end{array}$ & $\begin{array}{c}10 \\
\mathrm{mg} / \mathrm{kg} \\
\text { 5-o.-a. a. }\end{array}$ & $\begin{array}{c}100 \\
\mathrm{mg} / \mathrm{kg} \\
\text { 5-o.-a. a. }\end{array}$ & \\
\hline $\begin{array}{l}\text { Before } \\
\text { the diet }\end{array}$ & 134 & 111 & 71 & 66 & 41 & 36 & 23 & 32 & 15 \\
\hline $\begin{array}{c}\frac{1}{2} \mathrm{hr} \text {. after } \\
\text { the diet }\end{array}$ & 138 & 108 & 67 & 63 & 46 & 28 & 26 & 32 & 19 \\
\hline 1 & 144 & 85 & 78 & 65 & 45 & 31 & 26 & 32 & 14 \\
\hline 2 & 122 & 87 & 56 & 68 & 43 & 34 & 29 & 29 & 17 \\
\hline 3 & 125 & 107 & 63 & 53 & 41 & 39 & 23 & 37 & 17 \\
\hline 6 & 129 & 80 & 64 & 60 & 45 & 31 & 25 & 36 & 15 \\
\hline Average & 132 & 96 & 67 & 63 & 44 & 33 & 25 & 33 & 16 \\
\hline
\end{tabular}

\section{Discussion.}

Firstly, the secretory activity of the pancreatic calls presum $ə$ d from their minute structure will be discussed. For the sake of convenience in comparing the cell activi ty, 'production' and 'discharge' in the tables were manifestad in the figure. In the cases of administration of the diet after the X-ray irradiation (Fig. 4, 5, 6,7), both production and discharge of zymogen granules are extremely low, compared with those of the normal cass (Fig. 3). Production of zymogen granules is lowest in case of the irradiation of the epigastrium. In case of irradiation of the hypogastrium and the thigh, it seems to be a little more active than in the former case, but the differences as a matter of fact are almost negligible. Discharge of zymogen granules is a little more active than production of them, and especially in case of irradiation of the hypogastrium and the thigh (Fig. 6,7) this is plainly visible.

The quantity of the productin vacuoles in the gastric surface cclls in each case (obtained by KUSUMOTO) was described in the author's figure so that the relation between the sacretory activity of the pancreatic cells and the sacretion of productin might be clarified. It is clear that the səcretion of productin decreases a little after the X-ray irradiation and that the secretion of it is almost similar in all the cases of irradiation.

As the fact that production of zymogen granules is promoted by productin secreted has been previously proved, the extremely low production of zymogen granules mentioned above must be due to the inhibited action of the productin secreted. On the other hand, as the discharge of zymogen granules is considered to be initiated by secretin secretad from the duodenum, it is unrelated to productin. Nevertheless, it is noticed that the discharge is lowered as a result of the irradiation. However in this case the inhibitive effect of X-ray irradiation on the hypogastrium and the thigh is less than when irradiated on the epigastrium. This seems to be at variance with the fact that the inhibition of the action of productin has no relation with the area of irradiation. 


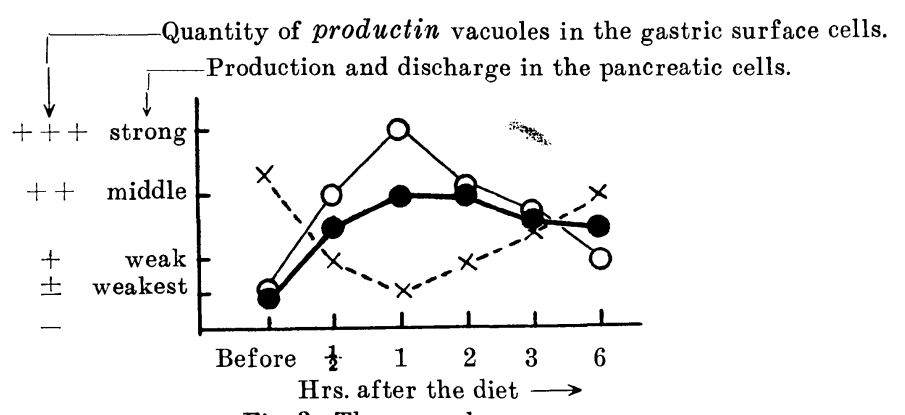

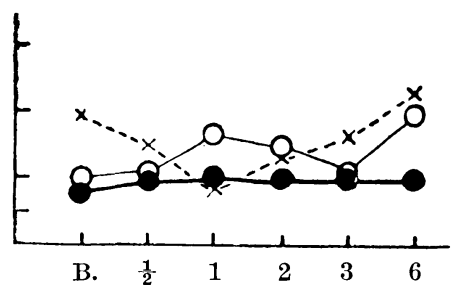

Fig. 4. $500 \mathrm{r}$ on the epigastrium.

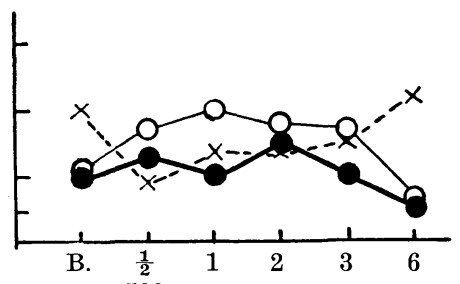

Fig. 6. $500 \mathrm{r}$ on the hypogastrium.

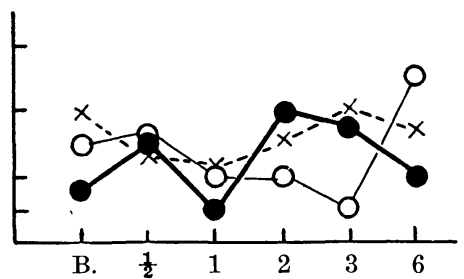

Fig. 8. The case of Pereston-N.

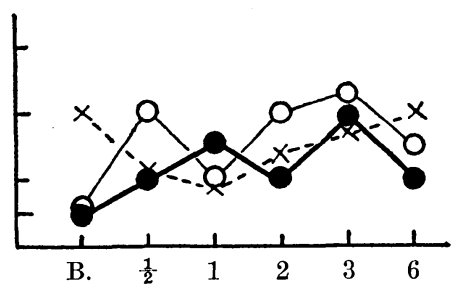

Fig. 10. The case of $10 \mathrm{mg} / \mathrm{kg} 5$-oxyanthranilic acid.

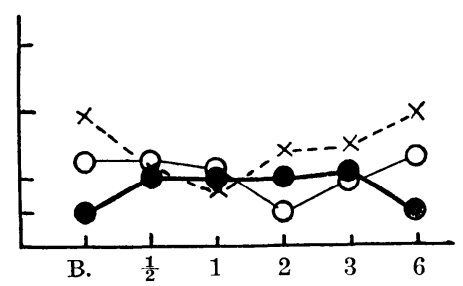

Fig. $5.100 \mathrm{r} \times 5$ on the epigastrium.

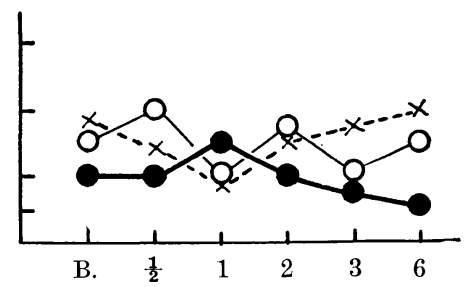

Fig. 7. $100 \mathrm{r} \times 5$ on the hypogastrium.

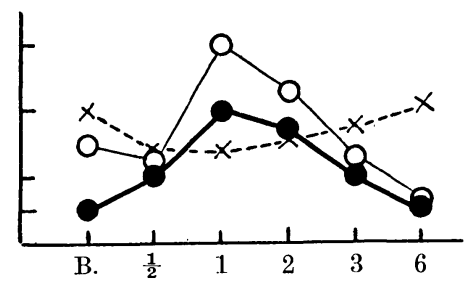

Fig. 9. The case of Thioctic acid.

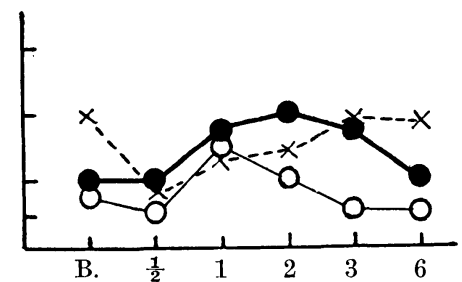

Fig. 11. The case of $100 \mathrm{mg} / \mathrm{kg} 5$-oxyanthranilic acid.

Fig. 3-11. - - production, - - - discharge of zymogen granules in the pancreatic cells. - - - the quantity of productin vacuoles in the gastric surface cells. 
In the results obtained from the experiments using chemical agents against $\mathrm{X}$-ray injuries (Fig. 8, 9, 10, 11), both the production and the discharge of zymogen granules are remarkably active. (Compare those with Fig. 4, 5, 6, 7.) Production and discharge of zymogen granules in case of thioctic acid, and production of them in case of $100 \mathrm{mg} / \mathrm{kg}$ 5-oxy-anthranilic acid are very similar to those of the normel case. This coincides with the results obtained from the secretory activity of the gastric peptic cells (KUSUMOTO). The secretion of productin in these cases is not different from that in the cases of irradiation without any agents so that the results are probably due to the inhibitive effects of these agents against the inhibitive effects of X-ray on the action of productin or secretin. The effects of each of the agents are different.

Secondly, a discussion of the results obtained from the histological observations: Except for an increase of the binucleate cells, no histological change in the pancreas can be seen.

As is pointed out by BÖHM, MÜNZER and PFUHL to count the binucleate cells in the sectioned specimens is not an accurate method. WADA has shown that the number of the binucleate liver cells obtained from the sectioned specimens of $7 \mu$ was only $47.6 \%$ of that obtained from the crushed specimens of the same liver. As the author used the sectioned specimens of $7 \mu$ stained by haematoxylin-eosin, the number of binucleate pancreatic cells must be small, but the comparison of each case may be possible.

The author's results differ considerably in each experiment as shown in Table 4. Viz. in cases of X-ray irradiation, the binucleate cells increase $4-8.5$ times the normal, and in case of the protective agents $1.6-2.8$ times. The rate of increase of number is in proportion to the rate of decrease of secretory activity. In detail, the number of the binucleate cells is the largest when the epigastrium is exposed, an average 132 cells can be counted in 1000 pancreatic cells after a single dose of $500 \mathrm{r}$ was given, and 96 cells after a daily dose of $100 \mathrm{r}$ for 5 days $(100 \mathrm{r} \times 5)$. When the hypogastrium and the thigh is exposed, the number decreases to almost half the former, viz. 67 cells after $500 \mathrm{r}$ and 63 cells after $100 \mathrm{r} \times 5$. The dose and the area of the irradiation may account for the difference. In other words, the effects of the $\mathrm{X}$-ray may reach the pancreatic cells directly. As mentioned above, the decrease of the secretory activity in the pancreatic cells takes place regardless of the area exposed, viz, between the secretory activity of the pancreatic cells and the X-ray irradiation, no direct relation can be seen. Therefore, binuclearity of the cell is considered to be unrelated to the secretory activity. OMOCHI et al. have presumed that the binuclearity of a cell must increase when the function of the cell is activated and WADA has suggested that the binuclearity of a cell is one of the living phenomena of the cell regardless of its function. The author's result confirms WADA's conclusion. This is more obvious when the effects of the protective agents are observed. Viz. when the agents are injected into abdominal cavity prior to the $500 \mathrm{r}$ irradiation on the epigastrium, the binucleate pancreatic cells do not increase more than 2.8 times the normal, and the more the inhibition of the secretory activity in the pancreatic cells decreases the more the decrease in the number of binucleate cells is seen. These findings prove that the binuclearity is not related to the secretory activity of the cell and that the chemical agents used are protective against the direct 
effect of X-ray on the cèll.

\section{Summary.}

As a part of the investigations on the effects of the X-ray irradiation on the digestive organs, its effects on the pancreas were studied. The X-ray was irradiated on the epigastrium or on the hypogastrium-thighs region of rats, a single dose being $500 \mathrm{r}$ and a daily dose being $100 \mathrm{r}$ for 5 days. The diet was given $1 \mathrm{hr}$. after the irradiation. The pancreas was taken and observed histocytologically before and $\frac{1}{2}$, $1,2,3,6$ hrs. after the diet. The results obtained may be summarized as follows.

1. The production of the zymogen granules in the pancreatic cells is extremely curtailed regardless of the dose and the area irradiated.

2. The discharge of the zymogen granules from the pancreatic cells is lowered remarkably after the irradiation on the epigastrium. It is less lowered after the irradiation on the hypogastrium-thighs.

3. When the protective agents against the X-ray injuries are injected into the abdominal cavity prior to the X-ray irradiation, the secretory activity of the pancreatic cells remains almost unchanged. The protective effect of thioctic acid and $100 \mathrm{mg} / \mathrm{kg} 5$-oxy-anthranilic acid on the production of zymogen granules, and the effect of thioctic acid on the discharge of them are easily discernible.

4. Binucleate pancreatic cells increase after the irradiation. They increase $6-$ 8.5 times in case of the irradiation on the epigastrium, 4 times in case of the hypogastrium-thighs region and 1.6-2.8 times in case of the injection of protective agents prior to the irradiation on the epigastrium. The number of binucleate calls decreases in proportion to the increase of the secretory activity of the pancreatic cells.

The author is grateful to Prof. K. OGASAWARA and Dr. T. KUSUMOTO in the Radiol. Dept. of Wakayama Med. College for their co-operation of X-ray irradiation.

内 容 自 抄.

X 線照射が消化系器官に及ぼす影響を檢する研究の一部として膵に及ぼす影響 を研究した。ラッテを用い 500 をを 1 回に，及び 1 日 100 とを 5 日連続して上腹 部並に下腹一大腿部に照射し, 照射後 1 時間を経て給食した。而して給食直前から 給食後 6 時間に亘って段階的に取材観察した。得た結果は以下の如くである.

1. 膵細胞に於けるチモーゲン顆䊀新生機能は照射量，照射部位に関係なく著 しく低下する。

2. チモーゲン顆粒放出機能は上腹部照射では甚だ低下するが，下㨫一大腿部照 射では前者よりあ活発である。照射量との関係は見出し難い，

3. X 線宿酔予防剂を照射前に注射しておくと，膵細胞分泌機能はあまり著㺫 には変化しない，チモーゲン顆䊀新生機能障害の防止には thioctic acid 及び5oxy-anthranilic acid が特に有效であり，チモーゲン顆粒放出機能障害の防止には thioctic acid が特に有效であると考えられる。

4. $\mathrm{X}$ 線照射例では 2 核性膵細胞が堌数する。救標本にて数えた結果では上 
腹部照射で正常の 6-8.5 倍, 下腹-大腿部照射で 4 倍, $\mathrm{X}$ 線宿酔予防剂注射後上 腹部照射で 1.6-2.8 倍で，分泌機能的活動が正常に近づくに従って減少する傾向 が罗出される。

\section{References.}

Böhm, J.: Untersuchungen über zweikernigen Zellen. Z. mikro.-anat. Forsch. 24 (1931). - Fujie, K. et al. : Cytological studies on the cause of pancreatic secretion. III. Knowleges on gastric hormones. (Jap. with Engl. abstr.) Arch. histol. jap. 3 (1952). - Experimental studies on the effects of surplus histamine to the pancreatic cells and the duodenal epithelial cells. (Jap. with Engl. abstr.) Arch. histol. jap. 10 (1956). - Furuta, K. : Study on the performing faculty of the secretory function in the pancreatic cells of rats fed with non-protein diet. Arch. histol. jap. 13 (1957). - Kusumoto, T.: Experimental study on the effects of X-ray on the secretory activity of the gastric chief cells. Arch. histol. jap. 20 (1960). Münzer, F. T.: Über die Zweikernigkeit der Leberzellen. Arch. mikro. Anat. 98 (1923). - Experimentelle Studien über die Zweikernigkeit der Leberzellen. Arch. mikro. Anat. 104 (1925). - Nomura, J.: The experimental studies on X-ray sensitivity of the pancreas. (Jap. with Engl. abstr.) J. of Radiol. phys. Ther. (Univ. Kanazawa) 44 (1958). - Omochi, S. et al. : On the relationship between cell function and binucleate cells. (Jap.) Acta anat. Nipp. 35 (1960). - Pfuhl, W.: Untersuchungen über zweikernige Zellen. Z. mikro.-anat. Forsch. 22 (1930). - Die mitotischen Teilungen der Leberzellen. Z. Anat. 109 (1939). - Wada, H. : Experimental studies on the binucleate liver cells. (Jap. with Engl. abstr.) J. Wakayama Med. Soc. 10 (1959). 